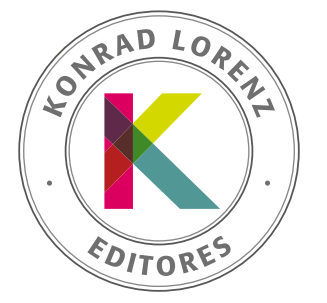

\title{
Efecto diferencial de un andamiaje metacognitivo en un ambiente e-learning sobre la carga cognitiva, el logro de aprendizaje y la habilidad metacognitiva
}

\author{
John Solórzano-Restrepo ${ }^{\mathrm{a}, \mathrm{b} *}$ y Omar López-Vargas
}

a Fundación Universitaria Konrad Lorenz, Bogotá, Colombia
${ }^{b}$ Universidad Pedagógica Nacional, Bogotá, Colombia

Recibido el 18 de octubre de 2018; aceptado el 1 de abril de 2019

\section{PALABRAS CLAVE}

Andamiaje metacognitivo, carga cognitiva, estilo cognitivo, logro de aprendizaje, conciencia metacognitiva

\section{KEYWORDS}

Metacognitive scaffolding, cognitive load, cognitive style, learning achievement, metacognitive consciousness
Resumen El presente estudio analiza los efectos generados por un andamiaje metacognitivo y el estilo cognitivo (dependiente/independiente de campo - DIC) en la carga cognitiva, la conciencia metacognitiva y el logro de aprendizaje cuando los estudiantes interactúan con un entorno de e-learning. Participaron 67 estudiantes de pregrado de la ciudad de Bogotá (Colombia), divididos en dos grupos. Uno interactuó con un entorno de aprendizaje con un andamiaje metacognitivo y el otro, sin él. Se utilizó la prueba EFT para establecer el estilo cognitivo de los aprendices y con la prueba MAI se determinó la conciencia metacognitiva. Se realizó un MANCOVA para analizar los datos. Los resultados sugieren diferencias entre la carga cognitiva intrínseca y extrínseca de los estudiantes dependientes de campo debido a la interacción entre el andamiaje metacognitivo y su estilo cognitivo. Los estudiantes que interactuaron con el andamiaje metacognitivo mostraron un mayor logro de aprendizaje y una mejor capacidad de monitoreo de su proceso de aprendizaje. (c) 2019 Fundación Universitaria Konrad Lorenz. Este es un artículo Open Access bajo la licencia CC BY-NC-ND (http://creativecommons.org/licenses/bync-nd/4.0/).

Differential effect of a metacognitive scaffolding in a e-Learning environment over cognitive load, learning achievement and metacognitive consciousness

Abstract The present study analyzes the effects generated by a metacognitive scaffolding and the cognitive style (Field Dependent/Independent-FDI) in the cognitive load, the metacognitive awareness and the achievement of learning when the students interact with an environment of e-Learning. 67 undergraduate students from the city of Bogotá (Colombia) participated, divided into two groups. One interacted with a learning environment with a metacognitive scaffolding and the other, without it. The EFT test was used to determine the cognitive style of the apprentices and the MAl test established the metacognitive awareness. Data were analyzed with a MANCOVA analysis. Results suggest differences between the intrinsic and extrinsic cognitive load in field dependent students due to the interaction between the metacognitive scaffolding

* Autor para correspondencia.

Correo electrónico: johnf.solorzanor@konradlorenz.edu.co 
and their cognitive style. Students who interacted with the metacognitive scaffolding showed a greater learning achievement and a better ability to monitoring their learning process. (c) 2019 Fundación Universitaria Konrad Lorenz. This is an open access article under the CC BYNC-ND license (http://creativecommons.org/licenses/bync-nd/4.0/).

Los ambientes de aprendizaje basados en la web se utilizan cada vez más en entornos educativos, para apoyar el proceso de enseñanza-aprendizaje en diferentes dominios de conocimiento y niveles de escolaridad. Estos ambientes tienen la promesa de lograr que los estudiantes participen de forma más activa en la construcción de su propio conocimiento, consiguiendo así un aprendizaje auténtico (Clark \& Mayer, 2008; Mayer, 2005; Shapiro, 2008). En los escenarios en línea los aprendices tienen la posibilidad de organizar en forma flexible su tiempo, al poder acceder a los contenidos desde cualquier lugar y momento (Jolliffe, Ritter \& Stevens, 2001; Song \& McNary, 2011).

A pesar de ello, algunos estudios muestran diferencias individuales en el uso efectivo y eficaz de los ambientes basados en la web, las cuales pueden estar relacionadas en forma directa con el estilo cognitivo, la carga cognitiva (CC) y la habilidad metacognitiva. Por ejemplo, respecto al estilo cognitivo, en la dimensión dependencia/independencia de campo (DIC), la generalidad de los estudios muestra que los estudiantes independientes de campo (IC) usan de forma más eficiente estos ambientes y obtienen mejores desempeños que sus compañeros dependientes de campo (DC) (Chen \& Macredie, 2004; Chou, 2001; Handal \& Herrington, 2004; López-Vargas, Ibáñez-Ibáñez \& Chiguasuque-Bello, 2014; López-Vargas, Sanabria-Rodríguez \& Sanabria-Español; 2014).

En cuanto a la CC, algunos trabajos evidencian que las características en el diseño instruccional pueden beneficiar o restringir el proceso de aprendizaje. En esta medida, el esfuerzo cognitivo que emplea un sujeto se puede ver afectado de forma negativa si la organización de la información no se presenta de forma adecuada; dificultando así la construcción de conocimiento (Clark \& Mayer, 2008; Mayer, 2005; Sweller, Ayres \& Kalyuga, 2011; Sweller, Van Merriënboer \& Paas, 1998).

Con relación a la habilidad metacognitiva, diferentes investigaciones muestran que cuando los estudiantes interactúan con escenarios de aprendizaje en línea tienen dificultades en el establecimiento de metas, no planean horarios y tiempos de estudio, no monitorean su aprendizaje $y$, en consecuencia, se les dificulta tomar decisiones para cambiar o ajustar las estrategias de aprendizaje (Azevedo, 2008; Lajoie \& Azevedo, 2006; Lehmann, Hähnlein \& Ifenthaler, 2014).

Algunos estudios muestran que el uso de andamiajes favorece el desempeño de los sujetos cuando se implementan en ambientes computacionales (Greene, Moos, Azevedo \& Winters, 2008; Kim \& Hannafin, 2011; Lehmann et al., 2014; Zhang, 2013); sin embargo, su uso puede estar asociado con variaciones en los niveles de carga cognitiva durante el aprendizaje, ya que esta puede relacionarse con el estilo cognitivo del sujeto, debido a que los estudiantes dependientes de campo son propensos a las distracciones y un andamiaje inadecuado podría convertirse en un distractor que aumente sus niveles.
Los andamiajes metacognitivos son un apoyo que se brinda al estudiante para que gestione y regule los procesos cognitivos; en ellos, el sujeto está en capacidad de autoimponerse metas, planear actividades, monitorear su ejecución y autoevaluar los resultados para cambiar o ajustar las falencias que se presentan (Molenaar, Boxtel \& Sleegers, 2010; Quintana, Zhang \& Krajcik, 2005; Zhang, 2013; Zhang \& Quintana, 2012). En este orden de ideas, la capacidad metacognitiva del aprendiz puede verse mejorada, por el uso de un andamiaje diseñado para favorecer la toma de decisiones frente a la organización, planeación, monitoreo y regulación del propio proceso de aprendizaje. Por tal motivo, el presente estudio plantea las siguientes preguntas de investigación:

¿Cuál es la influencia de un andamiaje metacognitivo sobre la carga cognitiva, la capacidad metacognitiva y el logro de aprendizaje de los estudiantes que construyen conocimiento a través de un ambiente en línea?

¿Existen diferencias significativas en la carga cognitiva, el logro de aprendizaje y la conciencia metacognitiva en estudiantes de diferente estilo cognitivo, en la dimensión dependencia/independencia de campo (DIC), cuando interactúan en un ambiente e-learning?

Dependencia/independencia de campo (DIC). Propuesto y desarrollado por Witkin y Goodenough (1981), es probablemente el estilo cognitivo más estudiado. En el ámbito de las tecnologías de la información aplicadas a la educación, estudios muestran de forma sistemática que los estudiantes IC, obtienen mejores desempeños que los DC. Los estudiantes DC prefieren contar con material de estudio organizado secuencialmente (lineal). En ambientes hipermedia se desorientan fácilmente, situación que les dificulta estructurar y reestructurar la información de forma eficaz; por tanto, prefieren que el proceso de navegación sea en grupo y guiado por otras personas (Chen \& Macredie, 2004; Handal \& Herrington, 2004; López-Vargas, Ibáñez-Ibáñez \& Racines-Prada, 2017).

En contraste, los ID prefieren libertad para navegar en el escenario y son capaces de establecer rutas de navegación organizadas. Usan de forma eficaz los recursos disponibles en el ambiente computacional y trabajan individualmente (Chen \& Macredie, 2004; Chou, 2001; López-Vargas et al., 2017).

Teoría de la carga cognitiva. Estudia la relación que existe entre la capacidad de memoria de trabajo y la construcción de conocimiento que logra un sujeto al interactuar con ambientes digitales. La CC se divide en tres clases: intrínseca $(\mathrm{Cl})$, extrínseca $(\mathrm{CE})$ y germánica $(\mathrm{CG})$. La $\mathrm{Cl}$ es inherente al tipo de tarea a desarrollar. Esta tiene en cuenta la dificultad del dominio de conocimiento a aprender y considera los conocimientos previos y almacenados en la memoria de largo plazo (Sweller, 2010). Por su parte, la CE se relaciona con la información dispuesta en el ambiente digital que no es relevante para el desarrollo de la tarea. Este tipo de carga está bajo el control del diseñador 
instruccional. Finalmente, la CG es la directamente responsable de la construcción de conocimiento. Permite crear o reestructurar los esquemas mentales y representa el logro real de aprendizaje (Andrade-Lotero, 2012; Van Merriënboer \& Sweller, 2005).

El desempeño académico se afecta cuando la estructura y organización de los contenidos digitales sobrecargan los limitados recursos de memoria del sujeto; por tanto, este no logra relacionar, de forma eficaz y eficiente, la información nueva con la almacenada en la memoria de largo plazo (Andrade-Lotero, 2012; Clark \& Mayer, 2008; Paas, Tuovinen, Tabbers \& Van Gerven, 2003; Sweller, 2010).

En este ámbito de investigación, el objetivo es reducir la CE y, de esta forma, aumentar el espacio en la memoria de trabajo para maximizar la CG (Cierniak, Scheiter \& Gerjets, 2009; Mayer \& Moreno, 2003; Paas, Renkl \& Sweller, 2003; Sweller, 2010). El uso de andamiajes dentro de la estructura de los ambientes computacionales, probablemente puede favorecer el aprendizaje y reducir la CE (Andrade-Lotero, 2012; López-Vargas et al., 2017).

La metacognición en el aprendizaje. Flavell (1976, 1979) la define como el conocimiento que tiene una persona acerca de sus propios procesos cognitivos y el control que puede ejercer sobre estos; es decir, es la capacidad que tienen las personas para gestionar y regular sus propios procesos de aprendizaje. Las investigaciones, en el contexto educativo, muestran sistemáticamente que las personas que despliegan habilidades metacognitivas tienen altas probabilidades de alcanzar las metas de aprendizaje y mejorar su desempeño académico (Hacker, Dunlosky \& Graesser, 2009).

En general, un aprendiz que posee habilidades metacognitivas es capaz de formularse metas, planear actividades, monitorear su desempeño durante la ejecución de estas, autoevaluarse continuamente, ajustar sus metas y valorar el resultado de su aprendizaje (Hernández-Barrios \& CamargoUribe, 2017; Zhang \& Quintana, 2012).

Andamiajes metacognitivos. Consisten en apoyos proporcionados dentro de un ambiente virtual, orientados a favorecer la planeación, el monitoreo, autoevaluación y control de los procesos cognitivos de manera consciente durante el desarrollo de tareas de aprendizaje (Kim \& Hannafin, 2011; Zhang \& Quintana, 2012). Al respecto, Quintana et al. (2005) y Molenaar et al. (2010) plantean que ayudan al estudiante a (1) planear lo que quiere aprender; (2) ejecutar y monitorear el avance de las metas, y (3) evaluar los resultados obtenidos para replantear las estrategias o metas impuestas.

\section{Método}

\section{Diseño}

La investigación es cuasiexperimental, con dos grupos de estudiantes de pregrado de la ciudad de Bogotá, Colombia. Como variable independiente se toma el ambiente e-learning con dos valores: grupo con andamiaje metacognitivo y grupo sin andamiaje. El estudio tiene una variable asociada: el estilo cognitivo en la dimensión DIC, con tres valores: dependientes, intermedios e independientes de campo. El diseño para el análisis de resultados es factorial $2 \times 3$.
Las variables dependientes del estudio son tres: logro de aprendizaje; carga cognitiva con tres valores: $\mathrm{Cl}$, CE y CG; $y$, finalmente, la conciencia metacognitiva con cinco valores: planificación (PL), organización (OR), monitoreo (MO), control (CO) y evaluación (EV). Como covariable se toma el logro de aprendizaje previo (calificaciones previas) y el estado inicial de su conciencia metacognitiva.

\section{Participantes}

En la investigación participaron de forma voluntaria 67 estudiantes de pregrado (61 mujeres y 6 hombres). La edad oscila entre 18 y 30 años $(M=20,24$ años, $D E=2.85)$.

\section{Instrumentos}

Cuestionario de carga cognitiva. Se empleó el cuestionario desarrollado por Leppink, Paas, Van Gog, Van der Vleuten y Van Merriënboer (2014), que permite identificar la percepción de los estudiantes sobre la carga cognitiva por medio de 13 ítems; Cl: ítems 1 a 4, CE: ítems 5 a 8, CG: ítems 9 a 13. El instrumento es un cuestionario de autorreporte y presenta una escala Likert de 0 a 10; donde 0 es completamente en desacuerdo y 10 completamente de acuerdo. El instrumento tuvo un alfa de Cronbach para la Cl de .751; para la CE de .760, y para la CG de .704. Los estudiantes respondieron el cuestionario cuatro veces (uno al final de cada unidad de aprendizaje).

Prueba de estilo cognitivo. Se utilizó el test de figuras enmascaradas (embedded figures test - GEFT) de Witkin para determinar el estilo cognitivo en la dimensión DIC. Se usó una versión en línea conservando los tiempos y las figuras presentes en la prueba original (Hederich-Martínez, López-Vargas \& Camargo-Uribe, 2016). La prueba mostró un alfa de Cronbach de .847. El promedio de la prueba EFT de la muestra fue de 8.94 ( $D E=3.94)$. Los estudiantes fueron agrupados en DC, intermedios e IC. Esto se hizo definiendo terciles para el puntaje total en la prueba (Angeli, 2013; Hederich-Martínez et al., 2016; Valencia-Vallejo, LópezVargas \& Sanabria-Rodríguez, 2019). Se identificaron tres rangos de puntajes: (a) 21 estudiantes DC (primer tercil), (b) 24 estudiantes intermedios (segundo tercil) y (c) 22 estudiantes IC (tercer tercil).

Logro de aprendizaje. Los estudiantes presentaron cuatro evaluaciones de 10 puntos de selección múltiple, contenidas en el ambiente e-learning (una por cada unidad de aprendizaje). Las evaluaciones tuvieron una fiabilidad alta, al presentar un alfa de Cronbach de .799.

Inventario de conciencia metacognitiva (MAI). Se empleó el test MAl (metacognitive awareness inventory) de Schraw y Moshman (1995), al inicio y al final de la investigación. Se tomaron 35 ítems para evaluar la regulación de la cognición, con cinco subcategorías, a saber: planificación, organización, monitoreo, control y evaluación. MAl es un cuestionario de autorreporte y presenta una escala Likert de 1 a 5 ; donde 1 es completamente en desacuerdo, y 5 completamente de acuerdo. Este instrumento se encuentra validado con estudiantes colombianos con un alfa de Cronbach de .94 (Huertas-Bustos, Vesga-Bravo \& Galindo-León, 2014). 
En esta investigación el instrumento tuvo un alfa de Cronbach de .92.

\section{Materiales}

Ambiente e-learning con andamiaje metacognitivo. Se empleó un ambiente e-learning desarrollado en el software Articulate Storyline, e insertado como paquete SCORM dentro de un LMS. El dominio de conocimiento fue teoría de la evolución, dividido en cuatro unidades de aprendizaje: historia, variación y herencia, selección natural, y selección sexual. Se implementó un andamiaje metacognitivo, el cual se fundamentó en el modelo propuesto por Winne (2001) y Hadwin y Winne (2001), con las siguientes características (véase figura 1).

Etapa 1. Presentación de la tarea de aprendizaje: el contenido general de cada módulo de aprendizaje se presenta al estudiante y se muestran los tiempos y espacios con que dispone para el desarrollo de las tareas.

Etapa 2. Planeación del aprendizaje: el aprendiz establece su meta de aprendizaje a través de un cuestionario que le permite establecer tiempos de estudio y los objetivos de aprendizaje para la unidad, en términos de una posible calificación dentro de una escala de 0 a 5 .

Etapa 3. Ejecución del plan de trabajo: el estudiante explora libremente los contenidos de cada unidad; y encuentra textos en los que, después de un tiempo, aparecen preguntas de reflexión: ¿Se está esforzando lo suficiente para entender los contenidos? ¿Cree que necesita tiempo adicional para entender este contenido? ¿El tiempo que planeó le está alcanzando?; recursos online adicionales para profundizar en los temas centrales; y autoevaluaciones para monitorear su aprendizaje.

Etapa 4. Resultados de aprendizaje: en esta etapa el aprendiz diligencia la evaluación final y realiza una reflexión alrededor de todo el proceso, a través de un cuestionario. Allí evalúa el nivel de logro alcanzado, la planeación de las actividades y la estrategia de aprendizaje seleccionada, mediante las siguientes preguntas: ¿Qué tanto aprendió usted sobre el tema de estudio? ¿Le pareció importante el contenido de este tema de estudio? ¿La meta de aprendizaje fue la más acertada? ¿El logro de aprendizaje obtenido fue el esperado?

\section{Procedimiento}

Para el desarrollo de la investigación se contactaron las directivas de la institución universitaria quienes aceptaron que los estudiantes de pregrado participaran en el estudio. Posteriormente, se les presentó la propuesta a los estudiantes y se les solicitó su consentimiento informado para su participación en el estudio; indicándoles que los resultados serían confidenciales y con fines investigativos. Finalmente, se procedió a aplicar el test EFT y el cuestionario MAI, de forma individual.

Se utilizaron dos versiones del ambiente e-learning, un curso de estudiantes accedió al ambiente que contenía el andamiaje metacognitivo, y el otro interactuó en el mismo

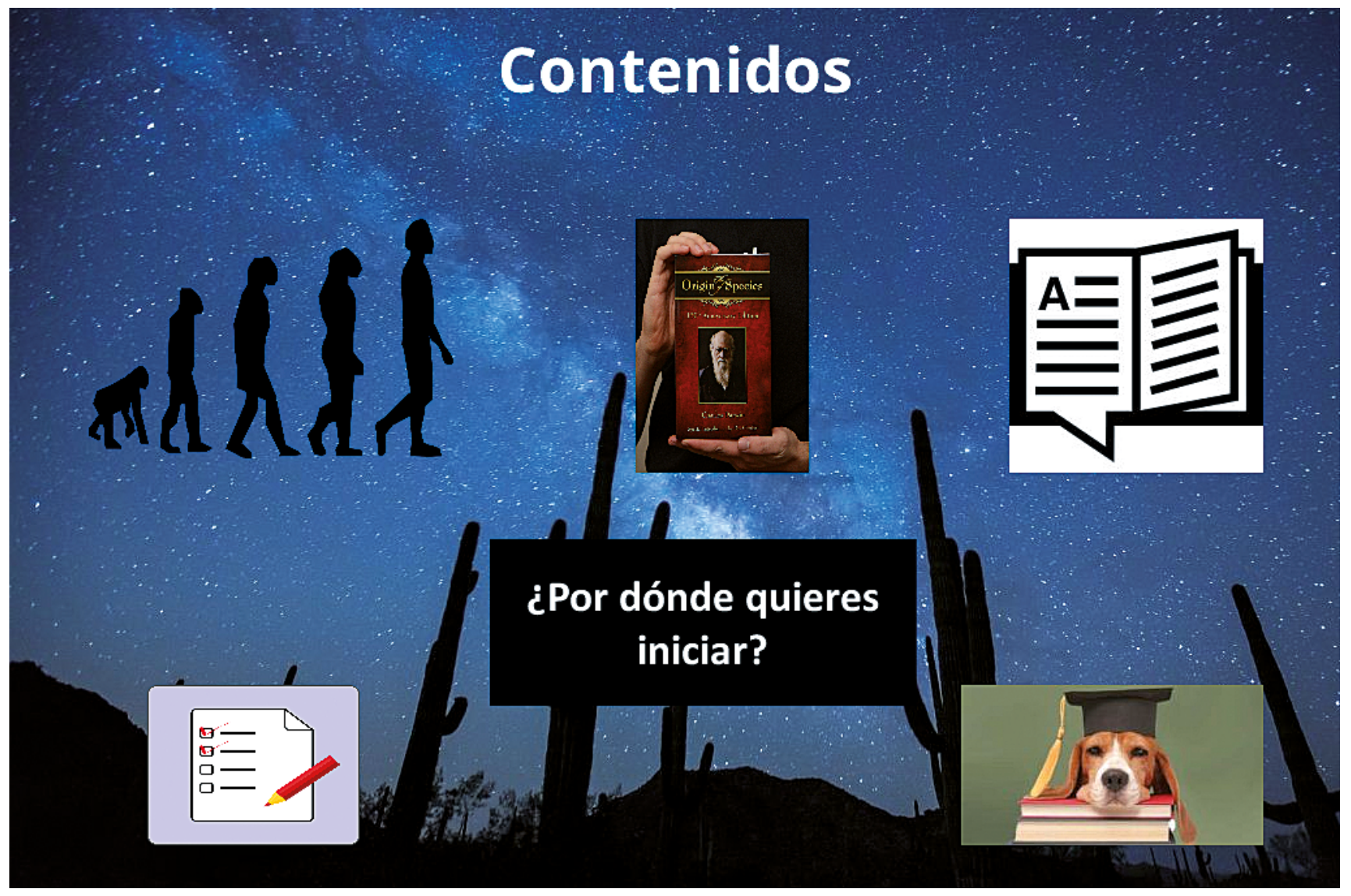

Figura 1 Interfaz del hipermedia sobre teoría de la evolución 
escenario, pero sin andamiaje. Les fue asignada una contraseña de identificación para acceder a los contenidos digitales y se distribuyó la temática para ser desarrollada en cuatro sesiones de trabajo de 90 minutos, una para cada unidad. Los datos se recolectaron a lo largo de tres semanas.

\section{Resultados}

Para el estudio de los resultados se utilizó un análisis factorial $2 \times 3$ de covarianza MANCOVA. Las variables dependientes del estudio fueron tres, a saber: la carga cognitiva con tres valores: $\mathrm{Cl}, \mathrm{CE}$ y $\mathrm{CG}$; el promedio del logro de aprendizaje; y la conciencia metacognitiva con cinco valores: PL, OR, MO, CO y EV.

En la tabla 1 se muestra un resumen de los estadísticos descriptivos de la carga cognitiva en los grupos de estudiantes que trabajaron con andamiaje y sin él, teniendo en cuenta su estilo cognitivo.

Efecto del andamiaje metacognitivo sobre el logro de aprendizaje, la carga cognitiva y la conciencia metacognitiva. Los resultados muestran diferencias significativas en el logro de aprendizaje de los estudiantes que trabajaron con el andamiaje $\left(F(1,54)=19.831, p<.001, \eta^{2}=.269\right)$. Los estudiantes que lo utilizaron mostraron mejores puntajes que los que trabajaron sin él. Frente a la CC los datos muestran que no existen diferencias significativas. No existen diferencias significativas por el uso del andamiaje sobre la $\mathrm{Cl}\left(F(1,54)=3.154, p=.081, \eta^{2}=.055\right)$, ni sobre la CE $(F$ $\left.(1,54)=3.188, p=.080, \eta^{2}=.056\right)$, y tampoco sobre la CG $\left(F(1,54)=.038, p=.846, \eta^{2}=.001\right)$. Finalmente, respecto a la conciencia metacognitiva no existen diferencias en PL $\left(F(1,54)=.321, p=.633, \eta^{2}=.004\right)$, en OR $(F(1,54)=.075$, $\left.p=.786, \eta^{2}=.001\right)$, ni en CO $\left(F(1,54)=.219, p=.642, \eta^{2}=.004\right)$, ni en $\operatorname{EV}\left(F(1,54)=.844, p=.362, \eta^{2}=.015\right)$. Solo se evidencian diferencias significativas en $M O(F(1,54)=7.364$, $\left.p=.009, \eta^{2}=.120\right)$.
Efecto del estilo cognitivo sobre el logro de aprendizaje, la carga cognitiva y la conciencia metacognitiva. Los resultados muestran que no existen diferencias sobre el logro de aprendizaje en atención a las diferencias individuales de acuerdo con el estilo cognitivo $(F(2,54)=2.575$, $\left.p=.086, \eta^{2}=.087\right)$. Tampoco existen diferencias significativas en cada tipo de CC. Finalmente, en las categorías del inventario de conciencia metacognitiva solo se observan diferencias significativas en $M O(F(2,54)=6.010, p=.004$, $\eta^{2}=.182$ ). Los estudiantes que usaron el andamiaje metacognitivo mostraron puntajes más altos.

Las comparaciones múltiples de acuerdo con Bonferroni indican que existen diferencias estadísticamente significativas $(p<.05)$ en el monitoreo solo entre estudiantes DC $(M=28.52, D E=4.11)$ e IC $(M=25.41, D E=4.43)$ (véase figura 2).

Efecto de la interacción entre el ambiente y el estilo cognitivo sobre el logro de aprendizaje, la carga cognitiva y la conciencia metacognitiva. Los resultados indican que no existen diferencias sobre el logro de aprendizaje en atención a la interacción entre el estilo cognitivo y el ambiente e-learning con y sin andamiaje $(F(2,54)=1.711$, $\left.p=.190, \eta^{2}=.060\right)$. Existen diferencias significativas en la CE $\left(F(2,54)=3.570, p=.035, \eta^{2}=.117\right)$. También existen diferencias significativas en la $\mathrm{Cl}(F(2,54)=3.314, p=.044$, $\left.\eta^{2}=.109\right)$. Finalmente, en las categorías del inventario de conciencia metacognitiva no se observan diferencias significativas por la interacción del estilo cognitivo y el ambiente e-learning. Las comparaciones múltiples de acuerdo con Bonferroni indican que existen diferencias estadísticamente significativas $(p<.05)$ en la CE entre los estudiantes $D C$ que no usan el andamiaje $(M=24.63, D E=5.45)$ con los dependientes que usan el andamiaje $(M=18.25, D E=6.19)$. También existen diferencias significativas en la $\mathrm{Cl}$ entre los estudiantes DC que no usan el andamiaje $(M=26.34, D E=$ $5.87)$ con los $D C$ que usan el andamiaje $(M=20.50, D E=3.15)$ (véase figura 3).

Tabla 1 Resultados de logro de aprendizaje, carga cognitiva y conciencia metacognitiva: las puntuaciones medias y desviaciones estándar entre paréntesis

\begin{tabular}{|c|c|c|c|c|c|c|c|c|c|c|c|}
\hline \multirow{2}{*}{$\begin{array}{l}\text { Ambiente } \\
\text { e-learning }\end{array}$} & \multirow{2}{*}{ Estilo cognitivo } & \multirow{2}{*}{$\mathbf{N}$} & \multirow{2}{*}{ Logro } & \multicolumn{3}{|c|}{ Carga cognitiva } & \multicolumn{5}{|c|}{ Conciencia metacognitiva } \\
\hline & & & & $\mathrm{Cl}$ & $\mathrm{CE}$ & CG & PL & OR & MO & CO & EV \\
\hline \multirow{4}{*}{$\begin{array}{l}\text { Con } \\
\text { andamiaje }\end{array}$} & $D C$ & 6 & $\begin{array}{l}35.54 \\
(3.88)\end{array}$ & $\begin{array}{l}20.50 \\
(3.15)\end{array}$ & $\begin{array}{l}18.25 \\
(6.19)\end{array}$ & $\begin{array}{l}29.07 \\
(5.26)\end{array}$ & $\begin{array}{l}27.00 \\
(4.60)\end{array}$ & $\begin{array}{l}41.83 \\
(4.96)\end{array}$ & $\begin{array}{l}32.33 \\
(1.75)\end{array}$ & $\begin{array}{l}21.33 \\
(2.16)\end{array}$ & $\begin{array}{l}22.83 \\
(3.25)\end{array}$ \\
\hline & Intermedio & 17 & $\begin{array}{l}36.13 \\
(5.55)\end{array}$ & $\begin{array}{l}19.76 \\
(2.86)\end{array}$ & $\begin{array}{l}18.63 \\
(3.79)\end{array}$ & $\begin{array}{l}27.66 \\
(4.18)\end{array}$ & $\begin{array}{l}24.59 \\
(3.94)\end{array}$ & $\begin{array}{l}38.35 \\
(4.74)\end{array}$ & $\begin{array}{l}27.65 \\
(3.18)\end{array}$ & $\begin{array}{l}20.06 \\
(3.36)\end{array}$ & $\begin{array}{l}20.53 \\
(4.08)\end{array}$ \\
\hline & IC & 11 & $\begin{array}{l}39.72 \\
(4.72)\end{array}$ & $\begin{array}{l}21.66 \\
(2.41)\end{array}$ & $\begin{array}{l}20.20 \\
(3.88)\end{array}$ & $\begin{array}{l}28.51 \\
(3.96)\end{array}$ & $\begin{array}{l}22.55 \\
(5.09)\end{array}$ & $\begin{array}{l}38.64 \\
(3.67)\end{array}$ & $\begin{array}{l}25.82 \\
(4.33)\end{array}$ & $\begin{array}{l}19.64 \\
(2.87)\end{array}$ & $\begin{array}{l}19.82 \\
(5.36)\end{array}$ \\
\hline & Total & 34 & $\begin{array}{c}37.19 \\
(5.22)\end{array}$ & $\begin{array}{l}20.51 \\
(2.83) \\
\end{array}$ & $\begin{array}{l}19.07 \\
(4.24) \\
\end{array}$ & $\begin{array}{l}28.18 \\
(4.21)\end{array}$ & $\begin{array}{l}24.68 \\
(4.47)\end{array}$ & $\begin{array}{l}39.06 \\
(4.52)\end{array}$ & $\begin{array}{l}27.88 \\
(4.01) \\
\end{array}$ & $\begin{array}{l}20.15 \\
(3.01) \\
\end{array}$ & $\begin{array}{l}20.71 \\
(4.60)\end{array}$ \\
\hline \multirow{4}{*}{$\begin{array}{l}\text { Sin } \\
\text { andamiaje }\end{array}$} & $\mathrm{DC}$ & 15 & $\begin{array}{l}28.33 \\
(5.61)\end{array}$ & $\begin{array}{l}26.32 \\
(5.87)\end{array}$ & $\begin{array}{l}24.63 \\
(5.45)\end{array}$ & $\begin{array}{l}29.72 \\
(4.43)\end{array}$ & $\begin{array}{l}25.07 \\
(4.10)\end{array}$ & $\begin{array}{l}39.60 \\
(5.08)\end{array}$ & $\begin{array}{l}27.00 \\
(3.78)\end{array}$ & $\begin{array}{l}19.87 \\
(2.33)\end{array}$ & $\begin{array}{l}21.40 \\
(2.92)\end{array}$ \\
\hline & Intermedio & 7 & $\begin{array}{l}33.07 \\
(5.19)\end{array}$ & $\begin{array}{l}31.39 \\
(5.80)\end{array}$ & $\begin{array}{l}19.96 \\
(4.40)\end{array}$ & $\begin{array}{l}27.31 \\
(5.31)\end{array}$ & $\begin{array}{l}25.14 \\
(4.74)\end{array}$ & $\begin{array}{l}39.57 \\
(2.82)\end{array}$ & $\begin{array}{l}26.14 \\
(3.72)\end{array}$ & $\begin{array}{l}20.43 \\
(1.90)\end{array}$ & $\begin{array}{l}21.43 \\
(1.90)\end{array}$ \\
\hline & IC & 11 & $\begin{array}{l}32.72 \\
(4.67)\end{array}$ & $\begin{array}{l}19.75 \\
(6.46)\end{array}$ & $\begin{array}{l}19.43 \\
(4.41)\end{array}$ & $\begin{array}{l}29.56 \\
(4.88)\end{array}$ & $\begin{array}{l}23.55 \\
(4.37)\end{array}$ & $\begin{array}{l}37.82 \\
(5.86)\end{array}$ & $\begin{array}{l}25.00 \\
(4.69)\end{array}$ & $\begin{array}{l}19.36 \\
(3.23)\end{array}$ & $\begin{array}{l}20.73 \\
(3.87)\end{array}$ \\
\hline & Total & 33 & $\begin{array}{l}30.80 \\
(5.56)\end{array}$ & $\begin{array}{l}23.08 \\
(6.62)\end{array}$ & $\begin{array}{l}21.91 \\
(5.40)\end{array}$ & $\begin{array}{l}29.16 \\
(4.59)\end{array}$ & $\begin{array}{l}24.58 \\
(4.25)\end{array}$ & $\begin{array}{l}39.00 \\
(4.92)\end{array}$ & $\begin{array}{l}26.15 \\
(4.06)\end{array}$ & $\begin{array}{l}19.82 \\
(2.54)\end{array}$ & $\begin{array}{l}21.18 \\
(3.03)\end{array}$ \\
\hline
\end{tabular}


Medias marginales estimadas de PosMonitoreo

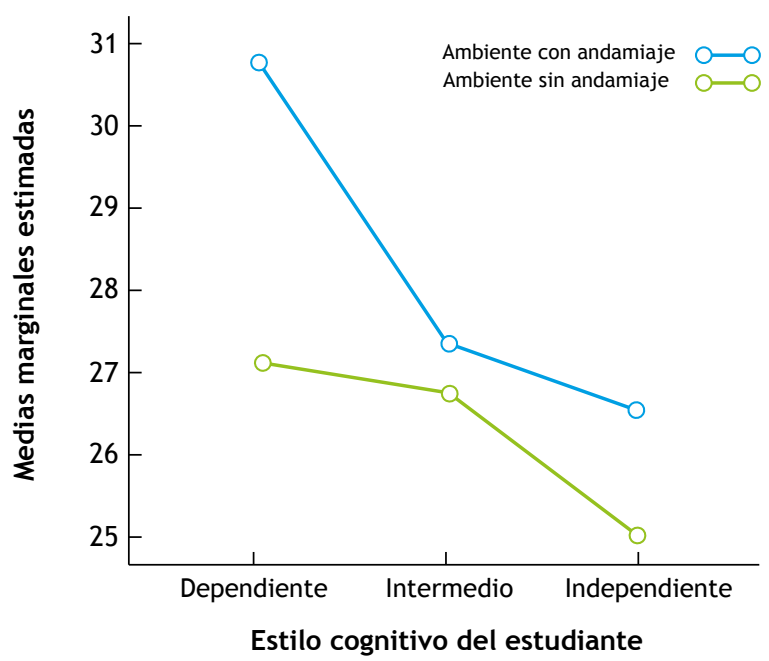

Las covariables que aparecen en el modelo se evalúan en los valores siguientes: PreCcondicional $=19.45$, PrePlanificación $=24.21$, PreOrganización $=38.16$, PreMonitoreo $=26.28$, PreControl $=20.27$, PreEvaluación $=20.30$, Logro Previo $=31.57$

Figura 2 Efecto de ambiente e-learning sobre el monitoreo

\section{Discusión}

Frente a la primera pregunta de investigación, los análisis del estudio indican que el grupo de estudiantes que usó el andamiaje obtuvo logros de aprendizaje significativamente mayores que sus compañeros que no lo usaron. Es posible afirmar que cada una de las etapas del andamiaje favorece el proceso de planeación, monitoreo, autoevaluación y control metacognitivo. Durante la ejecución del plan

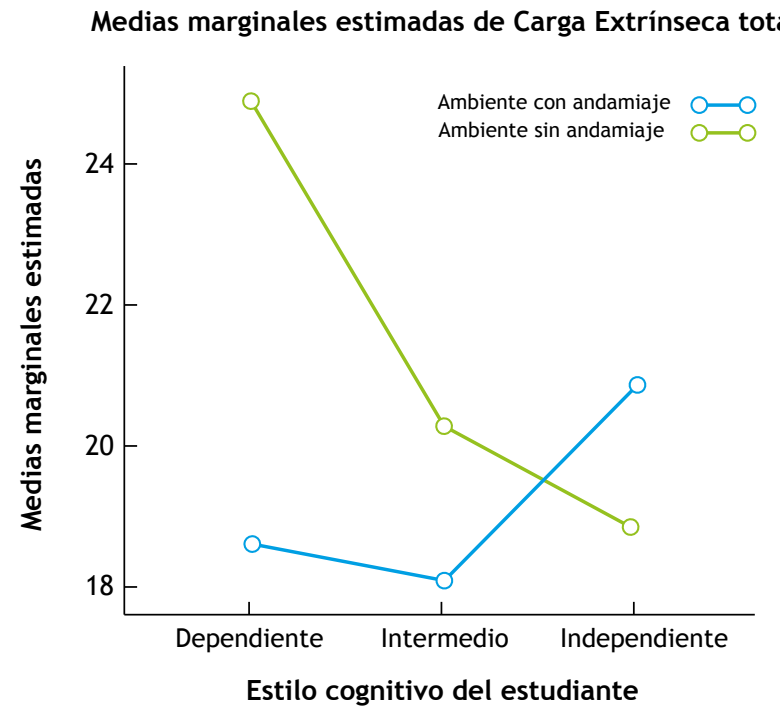

Las covariables que aparecen en el modelo se evalúan en los valores siguientes: PreCcondicional $=19.45$, PrePlanificación $=24.21$, PreOrganización $=38.16$, PreMonitoreo $=26.28$, PreControl $=20.27$, PreEvaluación $=$ 20.30, Logro Previo = 31.57 de estudio, el andamiaje ayuda a monitorear el proceso de aprendizaje por medio de activadores metacognitivos $y$ de un módulo de autoevaluación, el cual tiene por objetivo estimular la reflexión constante de lo aprendido, de lo que no entiende, y de lo que le hace falta por aprender.

Estos resultados son consistentes con otros estudios que indican que el uso de andamiajes metacognitivos favorece el desempeño académico de los estudiantes (Hederich-Martínez et al., 2016; Huertas-Busto, López-Vargas \& Sanabria-Rodríguez, 2017; Zhang \& Quintana, 2012). En este sentido, el andamiaje fomenta una conciencia metacognitiva y probablemente, el hecho de aprender de forma organizada y estratégica, favorece la construcción del propio conocimiento.

En cuanto a la CC, los resultados muestran que el andamiaje metacognitivo no redujo la percepción de esta en los estudiantes. Se esperaba la reducción de la CE con el uso del andamiaje de acuerdo con los resultados del estudio de López-Vargas et al. (2017); sin embargo, el andamiaje no tuvo ningún efecto sobre la CC. Esto es debido posiblemente a los sujetos del estudio que fueron estudiantes de pregrado, mientras que en el trabajo de López-Vargas et al. (2017), los participantes cursaban secundaria.

Aunque los resultados no son concluyentes, se debe seguir investigando con mayor profundidad; teniendo en cuenta que las particularidades de las poblaciones, el tipo de dominio de conocimiento, los conocimientos previos y las características de los ambientes digitales, entre otras, pueden afectar los resultados.

En los resultados obtenidos sobre la conciencia metacognitiva, se evidencia que el andamiaje mostró un efecto positivo sobre el MO del aprendizaje. Los resultados de la autoevaluación fueron un insumo para que los aprendices valoraran, de forma objetiva, su estado real de conocimiento y detectaran si la construcción que realizaban era superficial o profunda. Posiblemente, el MO permitió que los estudiantes lograran mejores resultados en su desempeño.

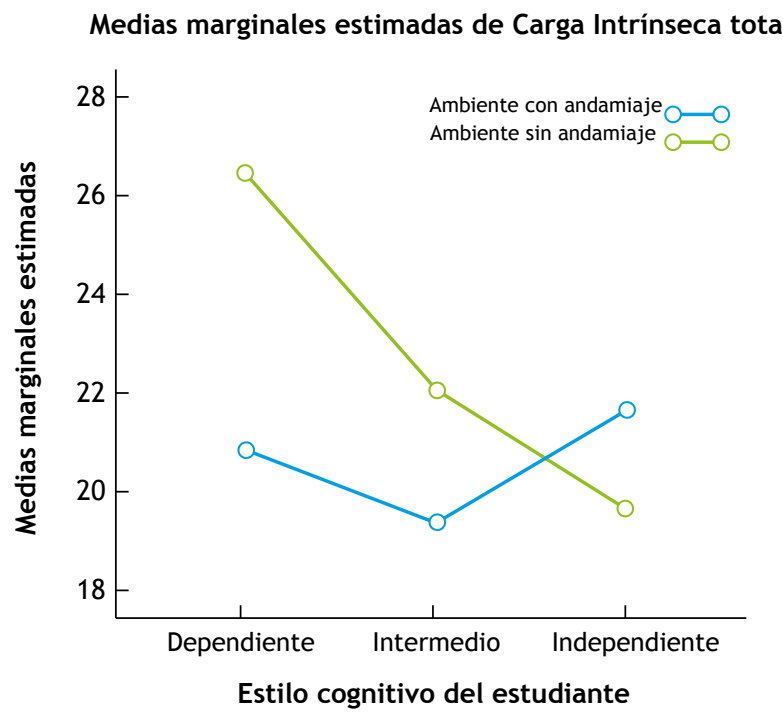

Las covariables que aparecen en el modelo se evalúan en los valore siguientes: PreCcondicional $=19.45$, PrePlanificación $=24.21$, PreOrgani zación $=38.16$, PreMonitoreo $=26.28$, PreControl $=20.27$, PreEvaluación 20.30, Logro Previo $=31.57$

Figura 3 Efecto de la interacción del estilo cognitivo y el ambiente e-learning sobre $\mathrm{CE}$ y $\mathrm{Cl}$ 
Algunos investigadores sostienen que supervisar el proceso de aprendizaje afecta de forma positiva el rendimiento académico (Dabarera, Renandya \& Zhang, 2014; Zhang \& Wu, 2009).

En este orden de ideas, la inclusión de módulos de autoevaluación en los ambientes digitales favorece el proceso de monitoreo del aprendizaje. Este mecanismo le permite al estudiante supervisar de forma objetiva el estado real de aprendizaje, evaluar y reflexionar sobre su proceso y tomar decisiones sobre "qué hacer" y "cómo hacerlo".

Con respecto a la segunda pregunta de investigación, los resultados muestran que no existen diferencias en el logro de aprendizaje entre los estudiantes de diferente estilo cognitivo. Es decir, los estudiantes DC e intermedios lograron aprendizajes equivalentes a los obtenidos por sus compañeros IC; resultados que complementan los hallazgos de otros estudios en donde se da cuenta que el uso de andamiajes, permiten reducir las diferencias individuales en el desempeño (López-Vargas \& Triana-Vera, 2013; LópezVargas et al., 2017; Martínez-Bernal, Sanabria-Rodríguez \& López-Vargas, 2016; Valencia-Vallejo, López-Vargas \& Sanabria-Rodríguez, 2018).

Respecto de la CC, los datos muestran que no existen diferencias significativas entre los estudiantes de diferente estilo cognitivo. Estos resultados coinciden con los hallazgos de Angeli (2013), quien no encontró diferencias de acuerdo con el estilo cognitivo de los aprendices. Sin embargo, los resultados del presente estudio contradicen los hallazgos de López-Vargas et al. (2017), quienes encontraron diferencias significativas entre estudiantes $D C$ e IC, en lo que respecta a la CC.

Estos resultados contradictorios sugieren que es necesario continuar con esta línea de investigación, pues no son claros los hallazgos frente a este tema. También es de indicar que la CC se mide con instrumentos de autorreporte, cuyas respuestas pueden incluir un sesgo de deseabilidad, y por tanto, tienen un alto componente subjetivo.

Ahora, frente a la conciencia metacognitiva, los resultados muestran diferencias significativas en los estudiantes con diferente estilo cognitivo durante el proceso de monitoreo del aprendizaje. Los DC muestran puntajes más altos que sus compañeros IC. De acuerdo con los resultados, el andamiaje fue efectivo para que los DC organizaran y ejecutaran, de forma sistemática, las diferentes actividades de aprendizaje.

Finalmente, los resultados muestran una interacción positiva entre el andamiaje y el estilo cognitivo del estudiante, lo cual favorece en forma positiva la reducción tanto de la $\mathrm{Cl}$ como de la $\mathrm{CE}$. Es decir, que aunque de manera independiente estas variables no afectaron la CC, su interacción favorece la disminución de estas cargas en los estudiantes DC.

Es posible que el uso del andamiaje refuerce la supervisión sistemática del propio proceso de aprendizaje y, en consecuencia, la percepción sobre la $\mathrm{Cl}$ y la $\mathrm{CE}$ del estudiante sea considerada menor, en comparación con el grupo de estudiantes DC que no utilizó el andamiaje. En cuanto a la CE, el andamiaje no presenta un esfuerzo adicional o un distractor que dificulte el aprendizaje de los contenidos temáticos (López-Vargas et al., 2017).

De acuerdo con la teoría de la CC y, específicamente, con la CE, era posible pensar que la inclusión del andamiaje afectaría de forma negativa la atención del estudiante en el tema de estudio, en la medida que las ventanas emergentes que se mostraban a los estudiantes podrían dividir la atención de estos, y teniendo en cuenta las características estilísticas de los estudiantes DC, era posible pensar que el andamiaje podría actuar como un distractor. Sin embargo, esto no se evidenció durante la investigación, pues se redujo la CE. Es viable pensar que favorecer la supervisión del propio proceso de aprendizaje por medio de andamiajes, les permite a los estudiantes dependientes de campo evaluar de forma objetiva el estado actual del proceso de construcción de conocimiento, y así cambiar o ajustar lo que sea necesario para lograr las metas autoimpuestas en la etapa inicial. Este proceso probablemente cambió la percepción del estudiante sobre el grado de dificultad de la tarea de aprendizaje; y así, el juicio sobre la $\mathrm{Cl}$ disminuyó también en ellos. Así las cosas, los resultados sugieren una correspondencia con el manejo eficiente de la memoria de trabajo de estudiantes DC a través de andamiajes que favorezcan el monitoreo de su proceso de aprendizaje.

Finalmente, los resultados sugieren que el uso de andamiajes puede ayudar a reducir las diferencias en el logro de aprendizaje en estudiantes de diferente estilo cognitivo en la dimensión DIC (López-Vargas et al., 2017; Valencia-Vallejo et al., 2019) al favorecer la reducción de la CC; sin embargo, es imprescindible explorar en mayor detalle sus características en atención a las diferencias individuales, para ser adaptables a las necesidades de aprendizaje de los estudiantes. En consecuencia, es necesario continuar desarrollando estudios que proporcionen evidencia empírica para que los diseñadores de escenarios digitales e investigadores en este ámbito desarrollen ambientes computacionales más equitativos y flexibles que favorezcan la autorregulación del proceso de aprendizaje.

De aquí surgen nuevos interrogantes acerca de cómo maximizar el aprendizaje y disminuir la CC de manera diferencial en estudiantes de diferentes niveles de escolaridad. Probablemente, el diseño de andamiajes sea una alternativa para que los estudiantes aprendan de forma más efectiva y utilicen la memoria de trabajo de manera eficiente a la hora de interactuar en ambientes digitales en la educación a distancia apoyada por ambientes web o tecnologías móviles.

\section{Notas de autor}

El presente manuscrito es derivado de la investigación realizada para optar por el título de Magíster en Tecnologías de la Información aplicadas a la educación en la Universidad Pedagógica Nacional.

\section{Referencias}

Andrade-Lotero, L. A. (2012). Teoría de la carga cognitiva, diseño multimedia y aprendizaje: un estado del arte [Cognitive load theory, design and multimedia learning: A state of the art]. Revista Internacional de Investigación en Educación, 5(10), 75-92.

Angeli, C. (2013). Examining the effects of field dependence-independence on learners' problem-solving performance and interaction with a computer modeling tool: Implications for the design of joint cognitive systems. Computers \& Education, 62, 221-230. http://dx.doi.org/10.1016/j.compedu.2012.11.002

Azevedo, R. (2008). The role of self-regulation in learning about science with hypermedia. In D. Robinson \& G. Schraw (Eds.), Recent innovations in educational technology that facilitate student learning (pp. 127-156). Charlotte, NC: Information Age Publishing.

Chen, S. Y., \& Macredie, R. D. (2004). Cognitive modeling of student learning in Web-based instructional program. International Journal of Human-Computer Interaction, 17(3), 375-402. http://dx.doi.org/10.1207/s15327590ijhc1703_5 
Chou, H. W. (2001). Influences of cognitive style and training method on training effectiveness. Computers and Education, 37, 11-25. http://dx.doi.org/10.1016/S0360-1315(01)00028-8

Cierniak, G., Scheiter, K., \& Gerjets, P. (2009). Explaining the split-attention effect: Is the reduction of extraneous cognitive load accompanied by an increase in germane cognitive load? Computers in Human Behavior, 25, 315-324. http://dx.doi.org/10.1016/j.chb.2008.12.020

Clark, R. C., \& Mayer, R. E. (2008). E-Learning and the science of instruction: Proven guidelines for consumers and designers of multimedia learning. San Francisco, CA: Pfeiffer /John Wiley \& Sons.

Dabarera, C., Renandya, W. A., \& Zhang, L. (2014). The impact of metacognitive scaffolding and monitoring on reading comprehension. System: An International Journal of Educational Technology and Applied Linguistics, 42(1), 462-473. http://dx. doi.org/10.1016/j.system.2013.12.020

Flavell, J. H. (1976). Metacognitive aspects of problem solving. In L. B. Resnick (Ed.), The nature of intelligence (pp. 231-236). Hillsdale, NJ: Lawrence Erlbaum.

Flavell, J. H. (1979). Metacognition and cognitive monitoring: A new area of cognitive-developmental inquiry. American Psychologist, 34(10), 906-911.

Greene, J. A., Moos, D. C., Azevedo, R., \& Winters, F. I. (2008). Exploring differences between gifted and grade-level students' use of self-regulatory learning processes with hypermedia. Computers \& Education, 50, 1069-1083. http:/dx./doi. org/10.1016/j.compedu.2006.10.004

Hacker, D. J., Dunlosky, J., \& Graesser, A. C. (2009). Handbook of metacognition in education. New York: Routledge.

Hadwin, A., \& Winne, P. (2001). CoNoteS2: A software tool for promoting self-regulation. Educational Research and Evaluation, 7, 313-334. http://dx.doi.org/10.1076/edre.7.2.313.3868

Handal, B., \& Herrington, T. (2004). On being dependent and independent in computer based learning environments. e-Journal of Instructional Science and Technology, 7(2), 1-10.

Hederich-Martínez, C., López-Vargas, O., \& Camargo-Uribe, A. (2016). Effects of the use of a flexible metacognitive scaffolding on self-regulated learning during virtual education, Int. J. Technology Enhanced Learning, 8(3/4), 199-216. http://dx.doi. org/10.1504/IJTEL.2016.082321

Hernández-Barrios, A., \& Camargo-Uribe, A. (2017). Adaptación y validación del Inventario de Estrategias de Autorregulación en estudiantes universitarios. [Validation of Self-Regulation Strategy Inventory Self-Report in university students]. Suma Psicológica, 24, 9-16. http://dx.doi.org/10.1016/j.sumpsi.2017.02.001

Huertas-Bustos, A., López-Vargas, O., \& Sanabria-Rodríguez, L. (2017). Influence of a metacognitive scaffolding for information search in B-learning courses on learning achievement and its relationship with cognitive and learning style. Journal of Educational Computing Research, 55(2), 147-171. http://dx.doi. org $/ 10.1177 / 0735633116656634$

Huertas-Bustos, A., Vesga-Bravo, G., \& Galindo-León, M. (2014). Validación del instrumento 'inventario de habilidades metacognitivas (MAl)' con estudiantes colombianos. [Validation of the instrument 'metacognitive awareness inventory (MAl)' with students in Colombia]. Praxis \& Saber, 5(10), 55-74. http://dx.doi. org/10.19053/22160159.3022

Jolliffe, A., Ritter, J., \& Stevens, D. (2001). The online learning handbook: Developing and using web-based learning. London: Kogan Page.

Kim, M. C., \& Hannafin, M. J. (2011). Scaffolding problem solving in technology-enhanced learning environments (TELEs): Bridging research and theory with practice. Computers \& Education, 56, 403-417. http://dx.doi.org/10.1016/j.compedu.2010.08.024

Lajoie, S. P., \& Azevedo, R. (2006). Teaching and learning in technology-rich environments. In P. A. Alexander \& P. H. Winne (Eds.), Handbook of educational technology ( $2^{\text {th }}$ ed.) (pp. 803821). Mahwah, NJ: Lawrence Erlbaum Associates.
Lehmann, T., Hähnlein, I., \& Ifenthaler, D. (2014). Cognitive, metacognitive and motivational perspectives on preflection in self-regulated online learning. Computers in Human Behavior, 32, 313-323. http://dx.doi.org/10.1016/j.chb.2013.07.051

Leppink, J., Paas, P., Van Gog, T., Van der Vleuten, C., \& Van Merriënboer, J. (2014). Effects of pairs of problems and examples on task performance and different types of cognitive load. Learning and Instruction, 30, 32-42. http://dx.doi.org/10.1016/j.learninstruc.2013.12.001

López-Vargas, O., \& Triana-Vera, S. (2013). Efecto de un activador computacional de autoeficacia sobre el logro de aprendizaje en estudiantes de diferente estilo cognitivo [Effect of a self-efficacy computational activator on learning achievement in students with different cognitive style]. Revista Colombiana de Educación, 64(1), 225-244. http://dx.doi.or$\mathrm{g} / 10.17227 / 01203916.64 \mathrm{rce} 225.244$

López-Vargas, O., Ibáñez-Ibáñez, J., \& Chiguasuque-Bello, E. (2014). El estilo cognitivo y la fijación de metas de aprendizaje en ambientes computacionales [Cognitive style and learning goals setting in computational environments]. Pensamiento Psicológico, 12(1), 133-148. http://dx.doi.org/10.11144/Javerianacali.PPSI121.ecfm

López-Vargas, O., Ibáñez-Ibáñez, J., \& Racines-Prada, O. (2017). Students' metacognition and cognitive style and their effect on cognitive load and learning achievement. Educational Technology \& Society, 20(3), 145-157.

López-Vargas, O., Sanabria-Rodríguez, L., \& Sanabria-Español, M. (2014). Logro de aprendizaje en ambientes computacionales: autoeficacia, metas y estilo cognitivo [Achievement of learning in computer environments: Self-efficacy, goals and cognitive style]. Psicología desde el Caribe, 31(3), 475-494. http://dx. doi.org/10.14482/psdc.31.3.5366

Martínez-Bernal, J., Sanabria-Rodríguez, L., \& López-Vargas, O. (2016). Relationships between learning achievement, self-monitoring, cognitive style, and learning style in medical students. Praxis \& Saber, 7(14), 141-164. http://dx.doi. org/10.19053/22160159.5221

Mayer, R. E. (2005). Cognitive theory of multimedia learning. In R. E. Mayer (Ed.), The Cambridge handbook of multimedia learning (pp. 31-48). Cambridge, UK: Cambridge University Press.

Mayer, R. E., \& Moreno, R. (2003). Nine ways to reduce cognitive load in multimedia learning. Educational Psychologist, 38(1), 43-52.

Molenaar, I., Van Boxtel, C., \& Sleegers, P. (2010). The effects of scaffolding metacognitive activities in small groups. Computers in Human Behavior, 26(6), 1727-1738. http://dx.doi.org/10.1016/j. chb.2010.06.022

Paas, F., Renkl, A., \& Sweller, J. (2003). Cognitive load theory and instructional design: Recent developments. Educational Psychologist, 38(1), 1-4. http://dx.doi.org/10.1207/S15326985EP3801_1

Paas, F., Tuovinen, J. E., Tabbers, H., \& Van Gerven, P. W. M. (2003). Cognitive load measurement as a means to advance cognitive load theory. Educational Psychologist, 38, 63-71. http://dx.doi.org/10.1207/S15326985EP3801_8

Quintana, Ch., Zhang, M., \& Krajcik, J. (2005). A framework for supporting metacognitive aspects of online inquiry through software-based scaffolding. Educational Psychologist, 40, 235244. http://dx.doi.org/10.1207/s15326985ep4004_5

Schraw, G., \& Moshman, D. (1995). Metacognitive theories. Educational Psychology, 7, 351-371.

Shapiro, A. (2008). Hypermedia design as learner scaffolding. Educational Technology Research and Development, 56, 29-44. http:// dx.doi.org/10.1007/s11423-007-9063-4

Song, L., \& MCNary, S. W. (2011). Understanding students' online interaction: Analysis of discussion board postings. Journal of Interactive Online Learning, 10(1), 1-14.

Sweller, J. (2010). Element interactivity and intrinsic, extraneous and germane cognitive load. Educational Psychology Review, 22, 123-138. http://dx.doi.org/10.1007/s10648-010-9128-5 
Sweller, J., Ayres, P., \& Kalyuga, S. (2011). Cognitive load theory. New York, NY: Springer.

Sweller, J., Van Merriënboer, J. J. G., \& Paas, F. G. (1998). Cognitive architecture and instructional design. Educational Psychology Review, 10(3), 251-296. http://dx.doi.org/10.1023/A:1022193728205

Valencia-Vallejo, N., López-Vargas, O., \& Sanabria-Rodríguez, L. (2018). Effect of a motivational scaffolding on e-learning environments: Self-efficacy, learning achievement, and cognitive style. Journal Educators On-line, 15(1), 1-14. http://dx.doi. org/10.9743/jeo2018.15.1.5

Valencia-Vallejo, N., López-Vargas, O., \& Sanabria-Rodríguez, L. (2019). Effect of a metacognitive scaffolding on self-efficacy, metacognition, and achievement in e-learning environments. Knowledge Management \& ELearning, 11(1), 1-19. http://dx.doi. org/10.34105/j.kmel.2019.11.001

Van Merriënboer, J. J. G., \& Sweller, J. (2005). Cognitive load theory and complex learning: Recent developments and future directions. Educational Psychology Review, 17(2), 147-177. http://dx.doi.org/10.1007/s10648-005-3951-0
Winne, P. H. (2001). Self-regulated learning viewed from models of information processing. In B. Zimmerman \& D. Schunk (Eds.), Self-regulated learning and academic achievement: Theoretical perspectives (pp. 153-189). Mahwah, NJ: Erlbaum.

Witkin, H. A., \& Goodenough, D. R. (1981). Cognitive Style: Essence and Origins. New York: International Universities Press.

Wu, H., \& Pedersen, S. (2011). Integrating computer- and teacher-based scaffolds in science inquiry. Computers \& Education, 57, 2352-2363. http://dx.doi.org/10.1016/j.compedu.2011.05.011

Zhang, L. J., \& Wu, A. (2009). Chinese senior high school EFL students' metacognitive awareness and use of reading strategies. Reading in a Foreign Language, 21, 37-59.

Zhang, M. (2013). Prompts-based scaffolding for online inquiry: Design intentions and classroom realities. Educational Technology \& Society, 16(3), 140-151.

Zhang, M., \& Quintana, C. (2012). Scaffolding strategies for supporting middle school students' online inquiry processes. Computers and Education, 58(1), 181-196. http://dx.doi.org/10.1016/j. compedu.2011.07.016 